\title{
Intermediate Band Solar Energy Conversion in ZnTeO
}

\author{
Jamie Phillips , Alan Teran , Chihyu Chen , E. Antolín - I. Ramiro , E. López , E. Hernández , I. Artacho , \\ C. Tablero, A. Martí , and A. Luque
}

\begin{abstract}
Energy conversion in solar cells incorporating ZnTeO base layers is presented. The $\mathrm{ZnTeO}$ base layers incorporate intermediate electronic states located approximately $0.4 \mathrm{eV}$ below the conduction band edge as a result of the substitution of $O$ in Te sites in the ZnTe lattice. Cells with $\mathrm{ZnTeO}$ base layers demonstrate optical response at energies lower than the ZnTe bandedge, a feature that is absent in reference cells with ZnTe base layers. Quantum efficiency is significantly improved with the incorporation of $\mathrm{ZnSe}$ emitter/window layers and transition from growth on GaAs substrates to GaSb substrates with a near lattice match to ZnTe.
\end{abstract}

Index Terms-Photovoltaic cells, heterojunctions, II-VI semiconductor materials, photoluminescence, oxygen.

\section{INTRODUCTION}

Intermediate energy states or energy bands in a semiconductor provide multiple spectral absorption bands that may be used to enhance solar energy conversion [1], [2]. However, efficient solar energy conversion requires proper positioning of the intermediate states to optimize spectral bands, strong optical transitions, and decoupling of thermal carrier processes in the intermediate states from the conduction and valence bands. Highly mismatched alloys (HMAs) are promising for intermediate band solar cells (IBSC), where the formation of intermediate states may be achieved and is often attributed to band anti-crossing behavior [3]. The use of an alloy system is attractive for an IBSC, offering the potential for a high density of states, strong optical absorption, and a production friendly process technology. ZnTe with dilute oxygen content is one such HMA, where the oxygen-tellurium substitution is known to provide electronic states $0.4 \mathrm{eV}$ below the conduction band [4]. Initial $\mathrm{ZnTeO}$ intermediate band solar cells have been demonstrated [5], [6], though device performance has been low due to challenges associated with junction formation and identification of a proper substrate for growth. In this work, the characteristics of solar cells with $\mathrm{ZnTeO}$ absorbers will be presented. Improved diode performance is achieved through epitaxial growth on GaSb substrates with a near lattice match and $\mathrm{n}$-ZnSe heterojunction window layers, as well as through use of graded ZnSeTe alloys. Subbandgap response is observed near $1.9 \mathrm{eV}$ corresponding to oxygen states in $\mathrm{ZnTe}$.

\section{EXPERIMENT}

Materials were grown by molecular beam epitaxy (MBE) using solid sources for $\mathrm{Zn}, \mathrm{Se}, \mathrm{Te}$, and a plasma source for oxygen. P-type ZnTe:N doping was achieved using a plasma

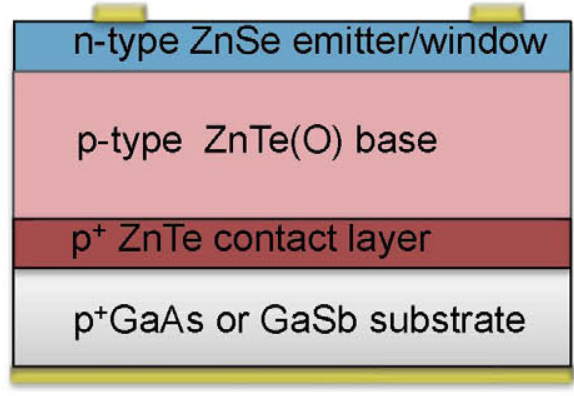

(a)

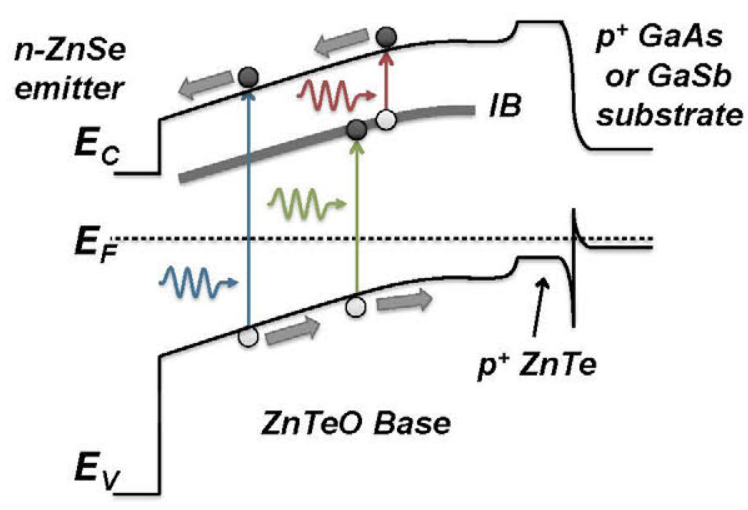

(b)

Fig. 1. Schematic diagrams of (a) solar cell device structure and (b) energy band diagram of the $\mathrm{ZnTeO}$ solar cell devices studied in this work.

source for nitrogen, and n-type $\mathrm{ZnSe}: \mathrm{Cl}$ doping was achieved using solid source $\mathrm{ZnCl}_{2}$. Epitaxial layers were grown on GaAs (001) and GaSb (001) substrates resulting in a lattice misfit with respect to $\mathrm{ZnTe}$ of $7.4 \%$ and $0.15 \%$, respectively. The structural and optical properties of the material were measured by $\mathrm{x}$-ray diffraction and photoluminescence spectroscopy.

Solar cells were fabricated using conventional photolithography, metallization, and etching techniques. The heterojunction material and device structure are shown in Fig. 1. The device structure consists of a heavily doped $\mathrm{p}-\mathrm{ZnTe}: \mathrm{N}$ contact layer, undoped $\mathrm{ZnTe}(\mathrm{O})$ base layer, and $\mathrm{n}-\mathrm{ZnSe}: \mathrm{Cl}$ emitter and window layer. Electrical characteristics were measured using a semiconductor device parameter analyzer. Optical 


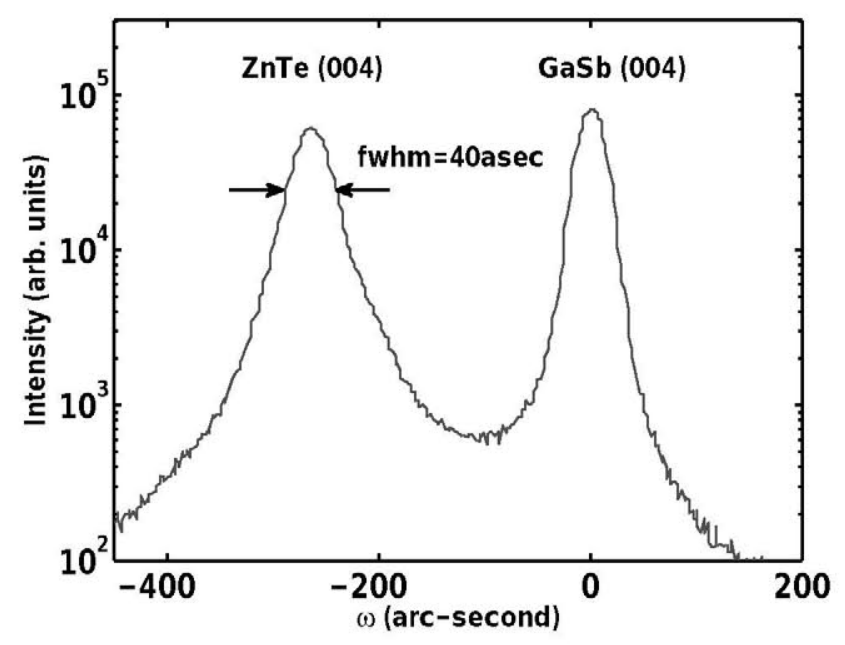

Fig. 2. X-ray diffraction $\omega-2 \theta$ scan for the (004) reflection of $\mathrm{ZnTeO}$ grown on $\mathrm{GaSb}(001)$.

measurements were performed using either a halogen lamp or calibrated AM1.5 source, as indicated in the text.

\section{RESULTS AND DISCUSSION}

\section{A. Material Properties}

The epitaxial materials demonstrate high quality structural and optical properties, with significantly improved behavior for $\mathrm{ZnTe}(\mathrm{O})$ grown on $\mathrm{GaSb}$ in comparison to $\mathrm{ZnTe}(\mathrm{O})$ grown on GaAs. Representative material properties are illustrated by the X-ray diffraction (XRD) and photoluminescence data in Fig. 2 and 3. The XRD $\omega-2 \theta$ scan shown in Fig. 2 illustrates good structural quality on $\mathrm{GaSb}$, with a full width at half maximum (fwhm) of 40 arcsec and a shift relative to the substrate of 264 arcsec corresponding to a lattice misfit of $0.1 \%$. Samples grown on GaAs exhibit broadened XRD peaks of several hundred arcsec due to an increase in dislocation density arising from the large lattice mismatch between $\mathrm{ZnTe}$ and GaAs.

Photoluminescence spectra for samples with varying oxygen flow rate are shown in Fig. 3. The maximum oxygen content corresponding to the $\mathrm{O}_{2}$ partial pressure of $10^{-5}$ Torr was determined to be approximately $0.5 \%\left(\mathrm{ZnTe}_{0.995} \mathrm{O}_{0.005}\right)$ based on independent SIMS and NRA measurements. The PL spectra at $\mathrm{T}=20 \mathrm{~K}$ for low oxygen flow demonstrate strong $\mathrm{ZnTe}$ bandedge emission near $2.38 \mathrm{eV}$ and emission from $O_{T e}$ substitutional impurities near $1.9 \mathrm{eV}$ [4]. The increase of oxygen flow rate results in an increase in the PL emission intensity and quenching of the ZnTe bandedge PL. The integrated PL intensity remains relatively unchanged, suggesting that the increase in oxygen content is not introducing any appreciable increase in non-radiative recombination pathways in the material. The quenching of the bandedge PL may be attributed to fast electron relaxation from the conduction band to the $O_{T e}$ states, as described from previous time-resolved optical measurements [7]. Optical absorption as a result of the $O_{T e}$ states has similarly been reported [8], and is the

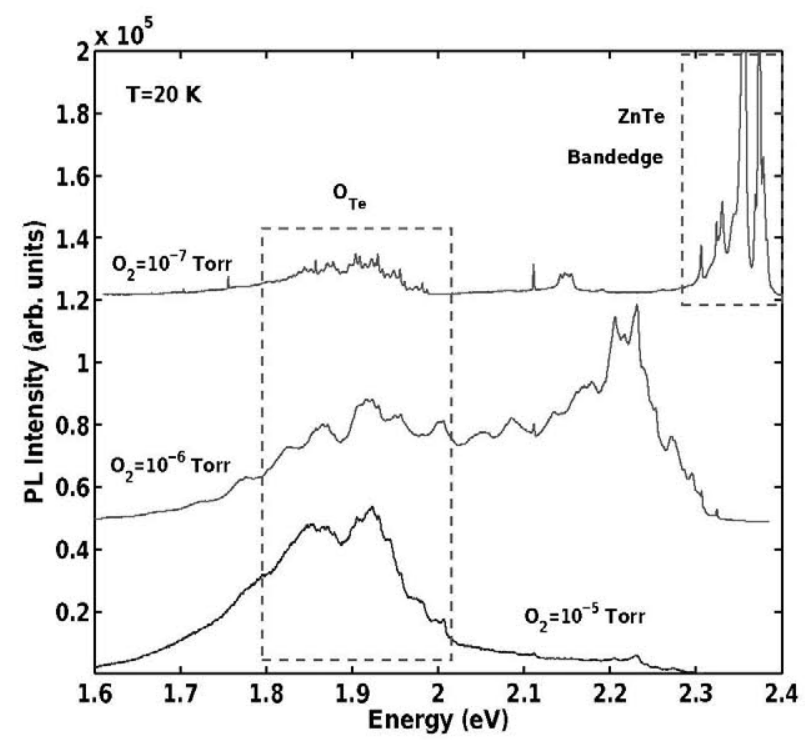

Fig. 3. Photoluminescence spectra of $\mathrm{ZnTeO}$ layers grown on $\mathrm{GaAs}$ under varying oxygen flow rate.

origin of intermediate band solar energy conversion. Temperature dependent photoluminescence measurements indicate an internal quantum efficiency of approximately $5 \%$, estimated from the ratio of PL emission at room temperature relative to low temperature.

\section{B. Current-Voltage Characteristics}

The $\mathrm{ZnTeO}$ solar cell devices exhibit strong rectifying behavior and photovoltaic response, as shown in the currentvoltage (J-V) characteristics in Fig. 4. Significant reduction in leakage current and enhancement in short circuit current are observed for samples grown on GaSb substrates. The short circuit current is improved by more than a factor of 10 using the same device structure on differing substrates. We believe that these improvements are primarily due to reduction in dislocation density, and therefore reduction in non-radiative recombination pathways in the material. The performance of these devices is still substantially below the ideal ZnTe diode, where an increase in open circuit voltage by approximately a factor of two can be expected for ideal material. The reduced open circuit voltage may be attributed to the highly-mismatched $\mathrm{p}-\mathrm{ZnTe} / \mathrm{n}-\mathrm{ZnSe}$ heterojunction, where a high dislocation density is expected for the lattice mismatch of approximately $7 \%$. Further improvements in open circuit voltage will require breakthrough technologies to achieve reliable n-type $\mathrm{ZnTe}$ and/or to significantly reduce the defect density at the p-ZnTe/n-ZnSe heterojunction interface.

\section{Spectral Response}

The influence of the intermediate band response cannot be effectively observed in the J-V characteristics due to variability in device performance that is likely due to variations in material quality and defect density. We have in fact observed all combinations of increased/decreased current 


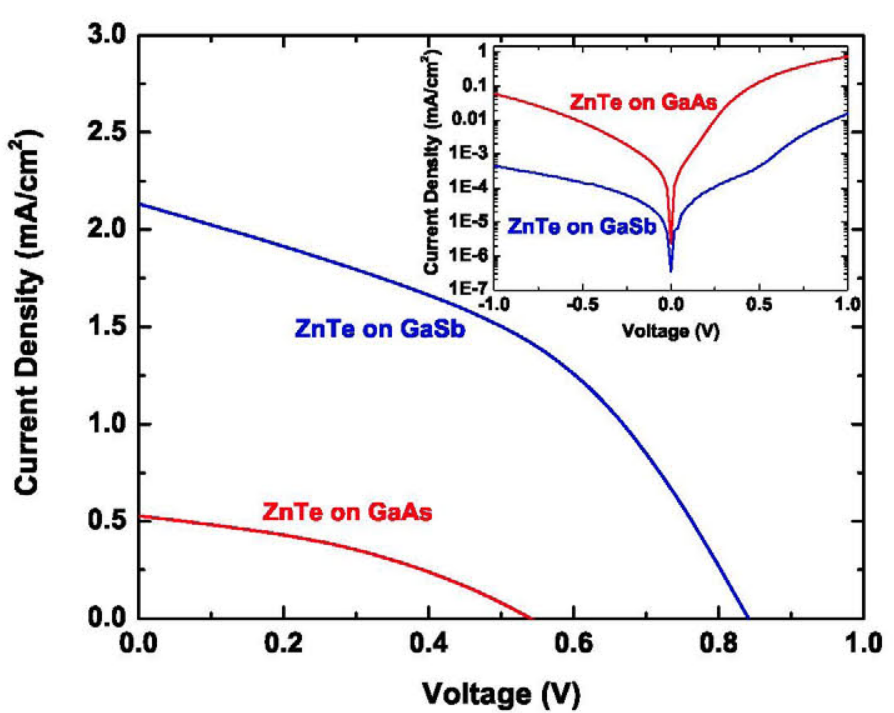

Fig. 4. Current-voltage response under illumination by a halogen lamp, comparing $\mathrm{n}-\mathrm{ZnSe} / \mathrm{p}-\mathrm{ZnTeO}$ heterojunction diodes on $\mathrm{GaAs}$ and $\mathrm{GaSb}$ substrates

and increased/decreased voltage when comparing cells with $\mathrm{ZnTeO}$ base layers to reference cells with $\mathrm{ZnTe}$ base layers. A more obvious means of detecting the intermediate band response is through the external quantum efficiency. Enhanced response is observed at wavelengths below the bandedge for cells with $\mathrm{ZnTeO}$ base layers, as shown in Fig. 5. The devices shown in Fig. 5 were taken from the same samples that were reported in 2009 [5] and measured two years later, illustrating the stability of the intermediate band transition over time. One feature of note in the quantum efficiency for the $\mathrm{ZnTeO}$ sample is the turn-on for the response corresponding to the ZnTe bandedge. In this device, the response is dominated by subbandgap processes. While the subbandgap response is desirably observed, the drop in response above ZnTe bandgap is sacrificed, suggesting a degradation in carrier transport and lifetime parameters that must be improved in order to make this material a viable technology.

Significant improvements in performance for response above the bandgap are realized through growth on $\mathrm{GaSb}$ substrates and incorporating an n-ZnSe emitter/window layer. The resulting $\mathrm{EQE}$ is shown in Fig. 6 for one of these devices and compared to the prior $\mathrm{p}-\mathrm{ZnTe} / \mathrm{n}-\mathrm{GaAs}$ reference cell in Fig. 5. An increase in the overall quantum efficiency is observed, likely due to reduced defect density and corresponding increase in minority carrier lifetime and minority carrier diffusion length. The $\mathrm{EQE}$ also maintains a relatively high value above the ZnTe bandedge, which can be attributed to the proper function of the $\mathrm{ZnSe}$ window layer in isolating the $\mathrm{ZnTe}(\mathrm{O})$ base layer from the surface. An inflection point and drop off in $\mathrm{EQE}$ is observed near $2.7 \mathrm{eV}$, corresponding to the $\mathrm{ZnSe}$ bandgap, further supporting this conclusion.

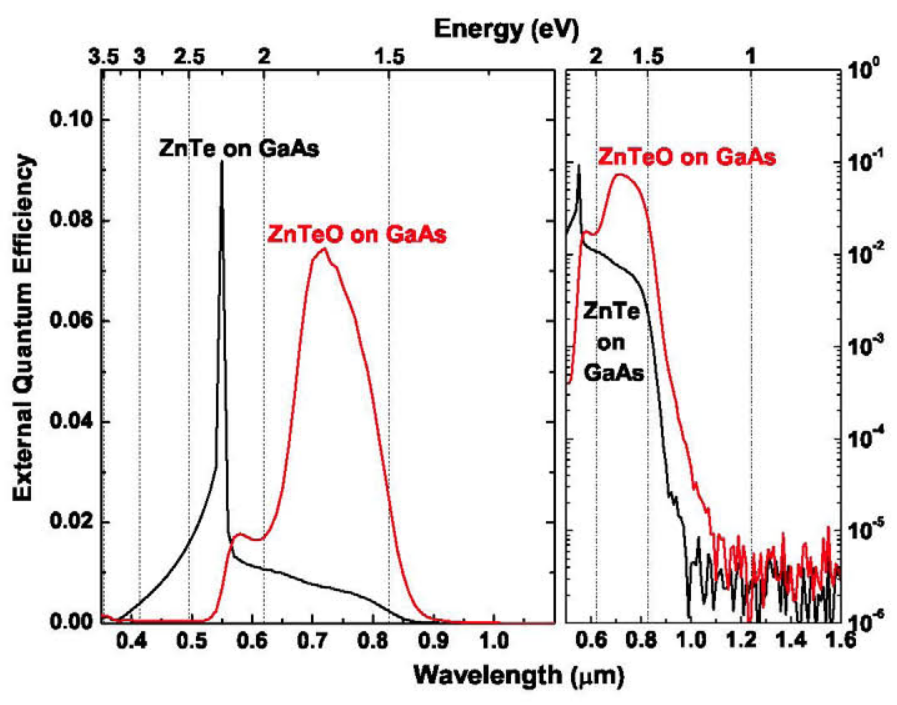

Fig. 5. External quantum efficiency of a p-Zn'Te(O) base layer on $n-G a A s$ demonstrating enhanced response below the $\mathrm{ZnTe}$ bandedge.

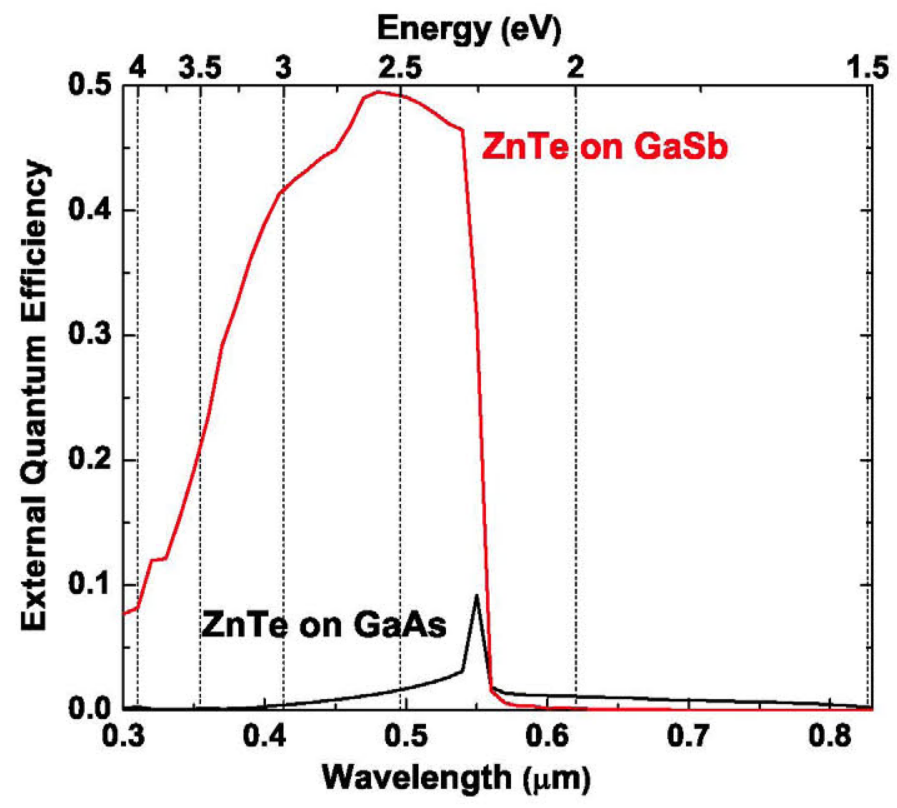

Fig. 6. External quantum efficiency comparing a p-ZnTe/n-GaAs solar cell on GaAs substrate to an $\mathrm{n}-\mathrm{ZnSe} / \mathrm{p}-\mathrm{ZnTe}$ solar cell on $\mathrm{GaSb}$ substrate

\section{Inverted p-ZnTe/ZnTeO/n-ZnSe Devices on GaAs}

One concern with the improved n-ZnSe/ZnTe(O)/p-ZnTe diodes is the location of the junction near the surface, which poses difficulty in carrier collection for subbandgap response where absorption is located further from the surface. An inverted device structure has been investigated that places the junction near the substrate interface, using an n-ZnSe buffer layer and intermediate graded n-ZnSeTe alloy, as shown in Fig. 7. The graded $\mathrm{ZnSeTe}$ layer is approximately $100 \mathrm{~nm}$ thick, and is intended to reduce dislocation formation in transitioning from the $\mathrm{ZnSe}$ lattice constant to the $\mathrm{ZnTe}$ lattice constant. The J-V characteristics of the inverted structure is shown 
in Fig. 8 in comparison to a representative $\mathrm{n}-\mathrm{ZnSe} / \mathrm{ZnTe} / \mathrm{p}$ $\mathrm{ZnTe} / \mathrm{GaSb}$ solar cell. One note on these measurements is the use of AM1.5 illumination for the data in Fig. 8, where photovoltaic response is lower than shown in Fig. 4 where halogen illumination is used due to the larger compenent of UV/visible light above the ZnTe bandgap for the halogen spectrum relative to AM1.5. The performance of the inverted structure with $\mathrm{ZnTeO}$ on GaAs actually exceeds the performance of the $\mathrm{ZnTe}$ on $\mathrm{GaSb}$, despite the large lattice mismatch for GaAs substrates and associated increase in dislocation density. This behavior demonstrates the importance of the $\mathrm{n}$-ZnSe/p-ZnTe junction in determining diode performance, where the graded $\mathrm{ZnSeTe}$ layer appears to be desirable for improving the electrical junction. Another key observation for the inverted diode structure is that the performance is maintained using a $\mathrm{ZnTeO}$ base layer, implying that the solar cell performance is not dramatically degraded by the presence of oxygen.

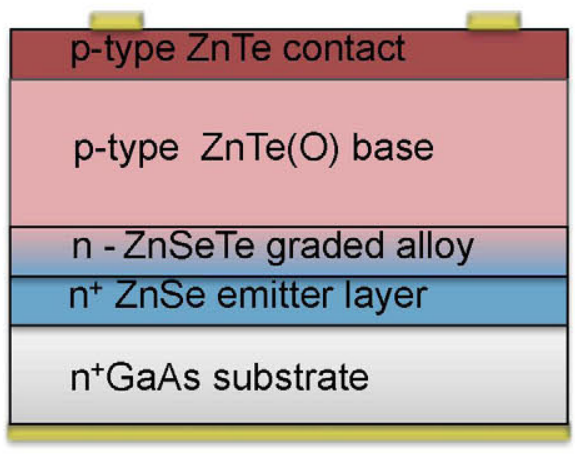

(a)

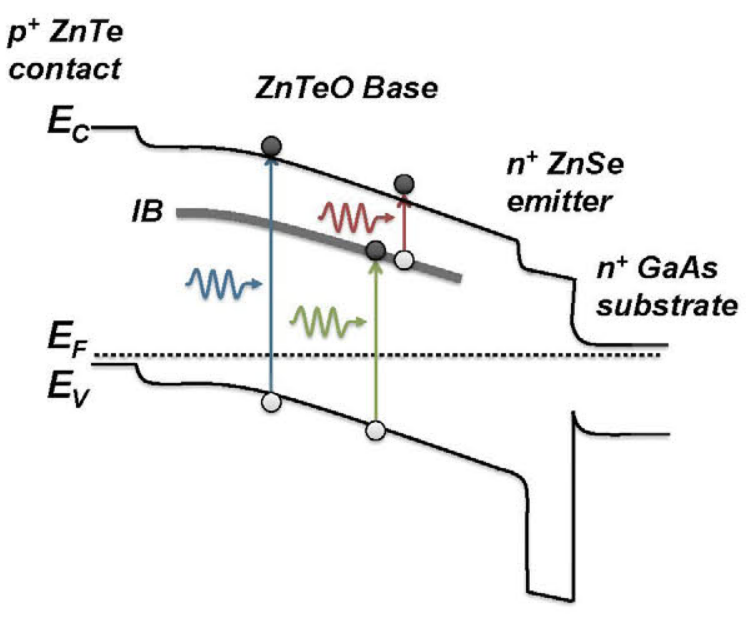

(b)

Fig. 7. Schematic diagrams of (a) solar cell device structure and (b) energy band diagram of the $\mathrm{ZnTeO}$ solar cell devices studied in this work.

Subbandgap response is also observed in the inverted structure, as demonstrated by the J-V characteristics in Fig. 9 with varying light excitation. Illumination with a red laser $(\lambda=635 \mathrm{~nm})$ and power density of $375 \mathrm{~mW} / \mathrm{cm}^{2}$ results in a photogenerated current and open circuit voltage of 0.3

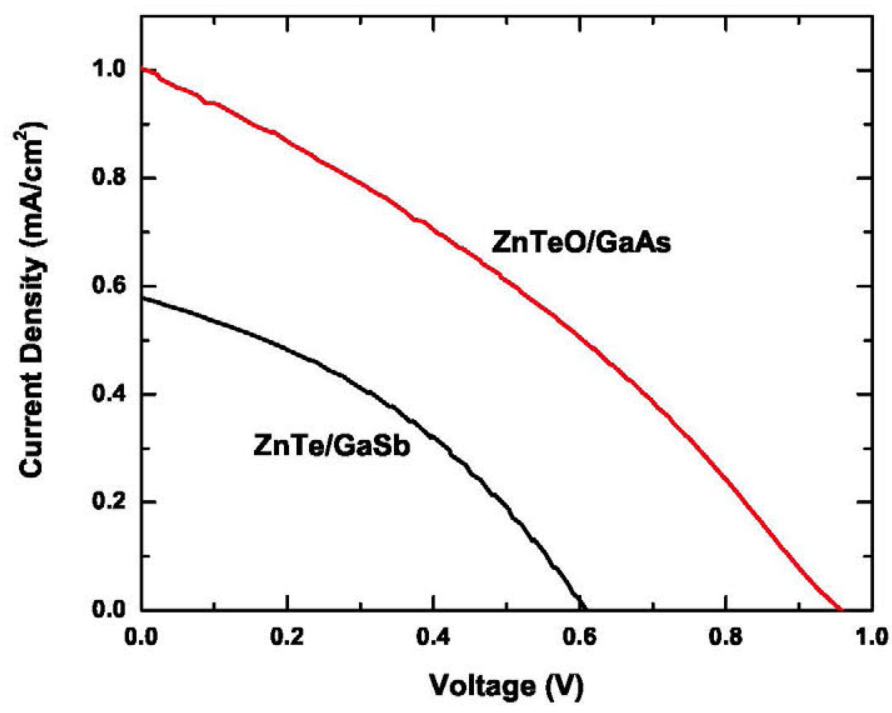

Fig. 8. Current-voltage response under AM1.5 illumination of inverted $\mathrm{p}-\mathrm{ZnTe} / \mathrm{ZnTeO} / \mathrm{n}-\mathrm{ZnSe} / \mathrm{GaAs}$ structure in comparison to $\mathrm{n}-\mathrm{ZnSe} / \mathrm{ZnTe} / \mathrm{p}$ $\mathrm{ZnTe} / \mathrm{GaSb}$.

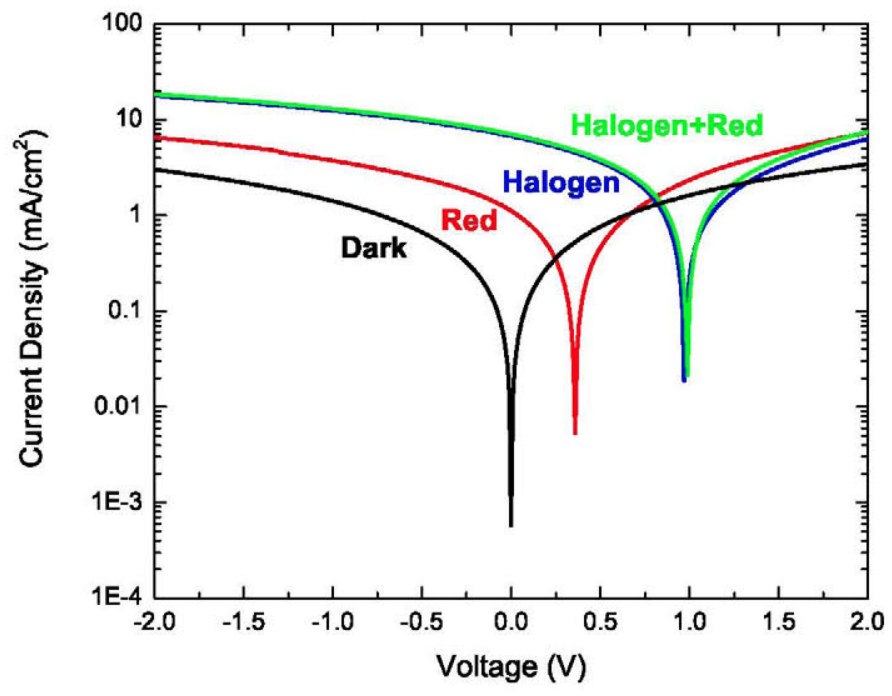

Fig. 9. Current-voltage characteristics of inverted $\mathrm{p}-\mathrm{ZnTe} / \mathrm{n}-\mathrm{ZnSe} / \mathrm{GaAs}$ diode with graded $\mathrm{ZnSeTe}$ layer comparing response under dark, subbandgap illumination with a red laser $(\lambda=635 \mathrm{~nm})$, halogen lamp, and halogen lamp with red laser.

V. In comparison to response with white light excitation with a halogen source, the addition of the red laser shows a small, but measurable, increase in response through addition contributions from subbandgap excitation.

\section{CONCLUSION}

The introduction of $O_{T e}$ substitutional impurities in $\mathrm{ZnTe}$ provides a path for sub-bandgap optical absorption without a significant sacrifice in the optical and electronic properties of the material. These transitions may be effectively utilized for an intermediate band solar cell material. Presently, the performance of solar cells utilizing $\mathrm{ZnTeO}$ base layers are limited by the ability to obtain a high-quality rectifying 
junction. The ability to grow on substrates with a near lattice match, the incorporation of wide-bandgap window layers, and graded ZnSeTe layers at the electrical junction have all shown improvement in the electrical performance of $\mathrm{ZnTe}$ heterojunction solar cells. An inverted diode structure on GaAs utilizing a $\mathrm{ZnTeO}$ base exhibits good performance relative to typical $\mathrm{ZnTe}$ heterojunction reference cells and demonstrate that oxygen does not dramatically decrease performance, while also providing subbandgap response to potentially increase conversion efficiency. Further improvements to produce a more ideal junction and to understand the energy conversion process associated with oxygen levels in ZnTe are critical in realizing the potential of ZnTeO intermediate band solar cells.

\section{ACKNOWLEDGMENT}

The collaborative experiments to study the $\mathrm{ZnTeO}$ solar cell device characteristics between UM and UPM was jointly funded by the National Science Foundation Materials World Network DMR-1006154 and the bilateral US-Spain Research Programme with Contract C11.0910B.01. Experiments at UM to study $\mathrm{ZnTeO}$ materials growth and optical properties were supported as part of the Center for Solar and Thermal Energy Conversion, an Energy Frontier Research Center funded by the U.S. Department of Energy, Office of Science, Office of Basic Energy Sciences under Award Number DE-SC0000957.

\section{REFERENCES}

[1] M. Wolf, "Limitations and possibilities for improvement of photovoltaic solar energy converters. I. Considerations for earth's surface operation", Proceedings of the Institute of Radio Engineers 48, 1246-1263 (1960).

[2] A. Luque and A. Marti, "Increasing the efficiency of ideal solar cells by photon induced transitions at intermediate levels", Physical Review Letters 78, 5014-17 (1997).

[3] K. M. Yu, W. Walukiewicz, J. Wu, W. Shan, J. W. Beeman, M. A. Scarpulla, O. D. Dubon and P. Becla, "Diluted II-VI oxide semiconductors with multiple band gaps", Physical Review Letters 91, 246403/1-246403/4 (2003).

[4] J. L. Merz, "Isoelectronic oxygen trap in $\mathrm{ZnTe",} \mathrm{Physical} \mathrm{Review} \mathrm{176,}$ 961-8 (1968).

[5] W. Wang, A. Lin and J. Phillips, "Intermediate-Band Photovoltaic Solar Cell Based on ZnTe:O", Applied Physics Letters 95, 011103 (2009).

[6] T. Tanaka, K. M. Yu, A. X. Levander, O. D. Dubon, L. A. Reichertz, N. Lopez, M. Nishio and W. Walukiewicz, "Demonstration of ZnTe1-xOx Intermediate Band Solar Cell", Japanese Journal of Applied Physics 50, 082304 (2011).

[7] W. Wang, A. Lin, J. D. Phillips and W. Metzger, "Generation and recombination rates at ZnTe:O intermediate band states", Applied Physics Letters 95, 261107 (2009)

[8] W. Wang, W. Bowen, S. Lin, S. Spanninga and J. Phillips, "Optical Characteristics of ZnTeO Thin Films Synthesized by Pulsed Laser Deposition and Molecular Beam Epitaxy", Journal of Electronic Materials 38, 119125 (2009). 